\title{
Plasma-laser ion discrimination by TOF technique applied to coupled SiC detectors.
}

\author{
Salvatore Cavallaro ${ }^{1,2,3}$ \\ ${ }^{1}$ Department of Physics and Astronomy, University of Catania, via S.Sofia,64 95123 Catania, Italy \\ ${ }^{2}$ LNS-INFN via S. Sofia, 6295123 Catania, Italy \\ ${ }^{3}$ CSFN\&SM via S.Sofia,64 95123 Catania, Italy
}

\begin{abstract}
The rate estimation of nuclear reactions induced in high intensity laser-target interaction $\left(\geq 10^{16} \mathrm{~W} / \mathrm{cm}^{2}\right)$, is strongly depending on the neutron detection efficiency and ion charge discrimination, according to particles involved in exit open-channels. Ion discrimination is basically performed by means of analysis of pits observed on track detector, which is critically dependent on calibration and/or fast TOF devices based on $\mathrm{SiC}$ and diamond detectors. Last setup is used to determine the ion energy and to obtain a rough estimation of yields. However, for each TOF interval, the dependence of yield from the energy deposited in the detector sensitive region, introduces a distortion in the ion spectra. Moreover, if two ion species are present in the same spectrum, the discrimination of their contribution is not attainable. In this paper a new method is described which allows to discriminate the contribution of two ion species in the wide energy range of nuclear reactions induced in laser-target interactions. The method is based on charge response of two TOF-SiC detectors, of suitable thicknesses, placed in adjacent positions. In presence of two ion species, the response of the detectors, associated with different energy losses, can determine the ion specific contribution to each TOF interval.
\end{abstract}

\section{Introduction}

With the availability of high intensities $\left(\geq 10^{16} \mathrm{~W} / \mathrm{cm}^{2}\right)$ laser-target interaction has evidenced nuclear events characterized by fast neutrons and/or recoiling ions involved in the induced specific reactions [1-8]. Estimated reaction rates and, consequently potential applications, are strongly depending on the neutron efficiency values and ion discrimination. Efficiencies of fast neutron detection are rather small, $10^{-4}$ to $10^{-3}$, depending on neutron energies [9-11]. Ion discrimination is performed with solid state nuclear track detectors (SSNTD), as CR-39 or PM-355, by analyzing the distribution of diameter etched pit observed into sample field of optical microscope. However the discrimination effectiveness is critically dependent on calibration (especially etching time), often coupled to thin foil degraders, or restricted to short energy range. [7],[12,13]. Furthermore atomic ion flux due to target impurities, which is expected much larger than nuclear one, can contribute to produce a false signal or, at least, an high statistical error. Thompson Spectrometer (TP), which could give almost complete ion discrimination $[14,15]$, presents the disadvantage of very small acceptance because of needed entrance-pinhole. On the other side, many attempts have been introduced in the past, to get more efficient CR-39 discrimination by coupling the diameter with other parameters (length
[16,17] ), (grey levels [18] ), with scarce experimental pursuance. TOF devices based on Silicon Carbide (SiC) and diamond detectors, almost insensitive to visible and near UV light, have been used to determine the possible energy of ions and to obtain a rough estimation of yields. An intrinsic problem can arise because of dependence of yields from energy deposited in the depleted region of detector subject to drifting electric field ( so-called active sample). In fact, yield for each time interval (named also time bin ), associated with sampling unit of TOF oscilloscope, depends on both number of ions arriving within and on energy deposited by each ion into active sample and converted in a corresponding number of electron-hole. The overall mechanisms and characteristics of charge drifting along the active electric field to the electrodes and finally converted in measured current-yield at readout circuit, are well described in many papers, as, for instance, in refs. [19,20]. Here it must be noticed that, because of energy dependence, the interpretation of measured yield as histogram height, is basically wrong. Moreover the active sample can represent full energy absorption for some ion and partial absorption (in punching through case) to others. In this paper, investigating on corrections needed to measured yields to obtain the effective distributions, a new method has been developed which, using correlated $\mathrm{SiC}$

Corresponding author: cavallaro@lns.infn.it 
detectors allows to discriminate the contribution of two ions in the wide energy range of nuclear reactions involved in laser-target interactions.

\section{Method description}

The method is based on the response of two TOF-SiC devices of appropriate depletion layers placed in adjacent positions and subjected to the simultaneous action of two species of ions produced by a plasmainduced nuclear reaction. In the absence of experimental data obtained in such conditions, the following procedure was referring to a nuclear reaction whose experimental data, obtained with a standard accelerator and ion beam hitting target, was as complete as possible. Thus, the available data was processed in such a way as to have the required characteristics.

To make the description of the method clear and effective, it is assumed an experimental setup similar to those commonly used today. The iodine laser system of PALS (Prague Laboratory), operating at single impulse at $1315 \mathrm{~nm}$, duration of $300 \mathrm{ps}$, is considered. Laser is supposed to irradiate in normal incidence a solid target where the generated plasma induces nuclear reactions. Several detectors are surrounding the target to monitoring the experiment in suitable pressure conditions ( $10^{-5}$ to $10^{-6} \mathrm{mbar}$ ). Two contiguous SiC-TOF devices are supposed backward at $\mathcal{Q}=60^{\circ}$ with respect to target normal. The TOF flight distance is $\mathrm{d}=1.25 \mathrm{~m}$. $\mathrm{SiC}$ devices are constituted by $2 \mathrm{SiC}$ diodes $1 \mathrm{~mm}^{2}$ in active surface and in adjacent position ( $1 \mathrm{~mm}$ far), depletion layer of $5 \dot{\mathrm{r}} \mathrm{m}$ and $80 \dot{\mathrm{m}} \mathrm{m}$, respectively.

The $\mathrm{SiC}$ detectors are generally built on a $4 \mathrm{H}-\mathrm{SiC}$ epitaxial layer, grown with the chemical vapor deposition technique in $\mathrm{HCl}$ atmosphere, which allows one to obtain a very thick depletion layer $(\sim 100 \mu \mathrm{m})$ with low dopant concentration and with very low defect concentration [19]. The Schottky diodes, reverse polarized and suitably equipped with an output-signal circuit, are coupled with a $50 \mathrm{Ohm}$ input resistor to a fast digital oscilloscope $(2 \mathrm{GHz}, 20 \mathrm{GS} / \mathrm{s})$. Typical active areas of detectors are $1 \mathrm{~mm}^{2}$ to $5 \mathrm{~mm}^{2}$, and depletion layer of $5 \dot{\mathrm{m}} \mathrm{m}$ to $\sim 100$ i $\mathrm{m}$ TOF time resolution of order of $1 \mathrm{~ns}$ can be easy achieved [20]. In the passing through the active layer, an ion spends almost fully the energy loss in pair electron-hole production and output signal formation. (e-hole pair $\mathrm{E} \cong 7.8 \mathrm{eV}$ ). When a flux of ions goes into the active area of detector simultaneously (i.e., into the time bin selected by TOF setup device), the associated charge depends on both the number of ions and the energy loss of ion into the active thickness of detector; which in turn depends on thickness and ion specie and energy.

\subsection{Ion discrimination in plasma-laser nuclear reactions}

Nuclear reactions produced in high intensity laser-target interaction involve, depending on atomic composition of the target, the simultaneous presence of different ion species, as proton, deuterons, ${ }^{3} \mathrm{He},{ }^{4} \mathrm{He}$, etc. Protons, in particular, are also present due to superficial impurities contained in targets. Typically, nuclear reactions, as $\mathrm{d}+$ $\mathrm{d}, \mathrm{d}+{ }^{3} \mathrm{He}, \mathrm{p}+{ }^{7} \mathrm{Li}, \mathrm{d}+{ }^{6} \mathrm{Li}, \mathrm{d}+{ }^{7} \mathrm{Li}, \mathrm{p}+{ }^{11} \mathrm{~B}$, etc, produce output ion energies of several $\mathrm{MeV}$ which can be mixed its self or mixed with impurity-protons. In presence of two ion species of $M_{1}$ and $M_{2}$ masses respectively, the TOF technique, for each time bin selects two mean energies, $E_{1}, E_{2}$ which are proportional to the ratio of masses $M_{1} / M_{2}$. So, for $p$ and $\alpha$, it will be $E_{\alpha}=4 \times E_{p}$. For example, in the $\mathrm{p}+{ }^{11} \mathrm{~B}$ reaction, $1 \mathrm{MeV}$ of proton can compete with $4 \mathrm{MeV}-\alpha$ which is rather a central energy value for last reaction (see later). In this and similar cases, the discrimination is needed.

\subsection{Simulation of TOF-SiC response and results}

Let one to consider, as a physical case, the reaction $\mathrm{p}+{ }^{11} \mathrm{~B}$, which has been object of many investigations in plasma-physics $[1,3,5,6,8]$.

At proton low energy $\left(\mathrm{E}_{\mathrm{p}}<3 \mathrm{MeV}\right)$ The exit open channels are:

1) $\mathrm{Be}^{8}(\mathrm{GS})+\alpha_{0},\left(\mathrm{Q}_{0}=8.59 \mathrm{MeV}\right)$ with ${ }^{8} \mathrm{Be}(\mathrm{GS})->2 \alpha$ by sequential decay, Q-value $=92 \mathrm{keV}$; and cross section ranging from 0.2 to 6 mbarn as proton energy varies from $\mathrm{Ep}=0.163 \mathrm{MeV}$ to $1.388 \mathrm{MeV}$.

2) $\mathrm{Be}^{8 *}\left(\right.$ excited state, $\left.\mathrm{E}^{*}=2.9 \mathrm{MeV}\right)+\alpha_{1}\left(\mathrm{Q}_{1}=5.69 \mathrm{MeV}\right)$ with cross section of 10-600-150 mbarn in the energy range $\mathrm{Ep}=0.165-0.600-1.388 \mathrm{MeV}$ with a resonance at $0.600 \mathrm{MeV}$.

3) ${ }^{12} \mathrm{C}+\gamma$ with cross section negligible with respect to previous channels.

4) ${ }^{11} \mathrm{~B}+\mathrm{p}$ elastic scattering with cross section decreasing from 500 to 200 barns with increasing energy of $0.3 \mathrm{MeV}$ to $1.5 \mathrm{MeV}$.

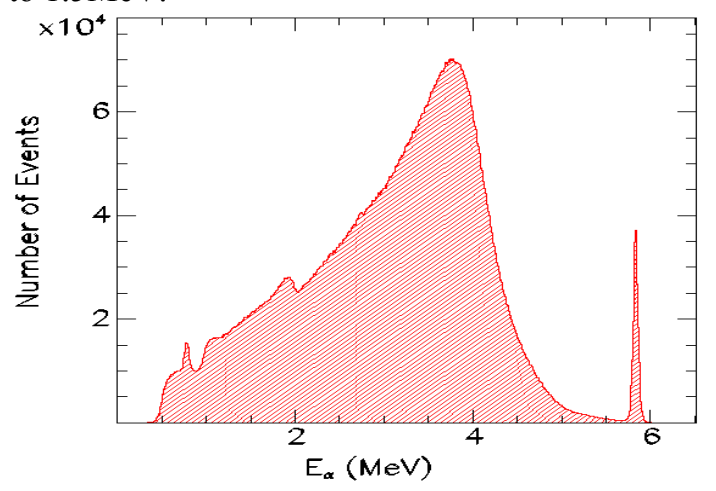

Fig. 1. Single-alpha Energy spectrum reproduced from ref. [21].

The overall contribution of channels 1 and 2 producing alpha particles, gives the energy single $\alpha$-spectrum shown in Figure 1. As reported in ref.[21], this spectrum has been measured with large area, two faced silicon detectors, equipped to measure $\alpha-\alpha$ coincidences. So, it can be considered as representative shaping of alpha energy spectrum. Taking into account of cross sections of channels 1 and 2, a rough estimation of alpha cross section can be of $\mathrm{d} \sigma / \mathrm{d} \omega \cong 230 \mathrm{mbarn} / \mathrm{sr}$. Elastic scattering contribution, as expected from Rutherford approximation, should be orders of magnitude higher. 
However, to make the basic conditions simpler (and clear the potentiality of the method), its contribution has been fixed to the same value assumed for alpha. To convert in TOF domain the energy spectra of proton and $\alpha$-particles, energy bins of $10 \mathrm{keV}$ and $0.10 \mathrm{~ns}$ time bins have been used, respectively. Figure 2 reports the proton elastic scattering, the $\alpha$ particles and the total $\mathrm{p}+\alpha$ contributions, as function of Energy (top part) and as function of TOF (bottom part), respectively. The proton and $\alpha$ yields are normalized to the integrated contributions. in energy ranges, by the expression:

$\sum_{E p m}^{E p x} Y p(E p) D E p=\sum_{E \alpha m}^{E \alpha x} Y \alpha(E \alpha) D E \alpha=230$ mbarn

where $\mathrm{Epm}=0.28 \mathrm{MeV}$ and $\mathrm{Epx}=1.5 \mathrm{MeV}$ are lower and higher limits of proton energy, $E \alpha m=0.30 \mathrm{MeV}$, $\mathrm{E} \alpha \mathrm{X}=5.99 \mathrm{MeV}$ the corresponding values for $\alpha$-particle and $\mathrm{DEP}=\mathrm{DE} \alpha=10 \mathrm{keV}$ the energy bins.
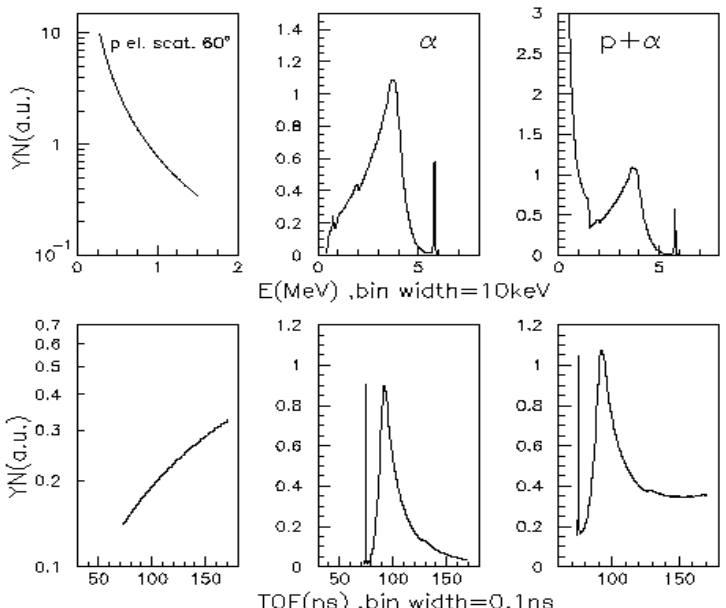

Fig.2. $p+{ }^{11} B$ reaction contributions for $p, \alpha$ and total $p+\alpha$, to Energy (top) and TOF spectra (bottom), respectively. TOF spectra have been calculated in the interval 73.3-170ns with time bin of $0.10 \mathrm{~ns}$.

Now, by assuming these contributions as representative of data obtainable with discriminative detectors, we regard to the signal amplification factors related to energy losses in the depletion layer of detectors.

Taking into account these factors, the TOF spectra of Figure 2 are modified as shown in Figure 3, for devices $\mathrm{SiC} 5 \mu \mathrm{m}$ (top) and $\mathrm{SiC} 80 \mu \mathrm{m}$ (bottom). As it will be shown, last $(p+\alpha)$ spectra can be considered as simulated response of $\mathrm{SiC} 5 \mu \mathrm{m}$ (top) and $\mathrm{SiC} 80 \mu \mathrm{m}$ (bottom), respectively.

Let us consider a normalized yield $\Delta \mathrm{Y}_{\mathrm{t}}$, at time bin $\Delta \mathrm{T}$ centered at time $\mathrm{T} i$ were contributions of both protons and alpha particles are present:

We have:

$$
\underset{t}{\Delta Y_{p}}=\Delta Y_{\alpha}+\Delta Y_{p}
$$

with $\Delta \mathrm{Y}_{\mathrm{p}}=\mathrm{Y}_{\mathrm{p}}(\mathrm{Ti}) \Delta \mathrm{T}$ and $\Delta \mathrm{Y}_{\alpha}=\mathrm{Y}_{\alpha}(\mathrm{Ti}) \Delta \mathrm{T}$ (3)
To $\mathrm{Ti}$ correspond Energy values $\mathrm{E}$ and $\mathrm{E}$ as the ratios

$E_{p} / E_{\alpha}=M_{p} / M_{\alpha}$ and energy losses in the active region of detector $\Delta \mathrm{E}_{\mathrm{p}}, \Delta \mathrm{E}_{\alpha}$.

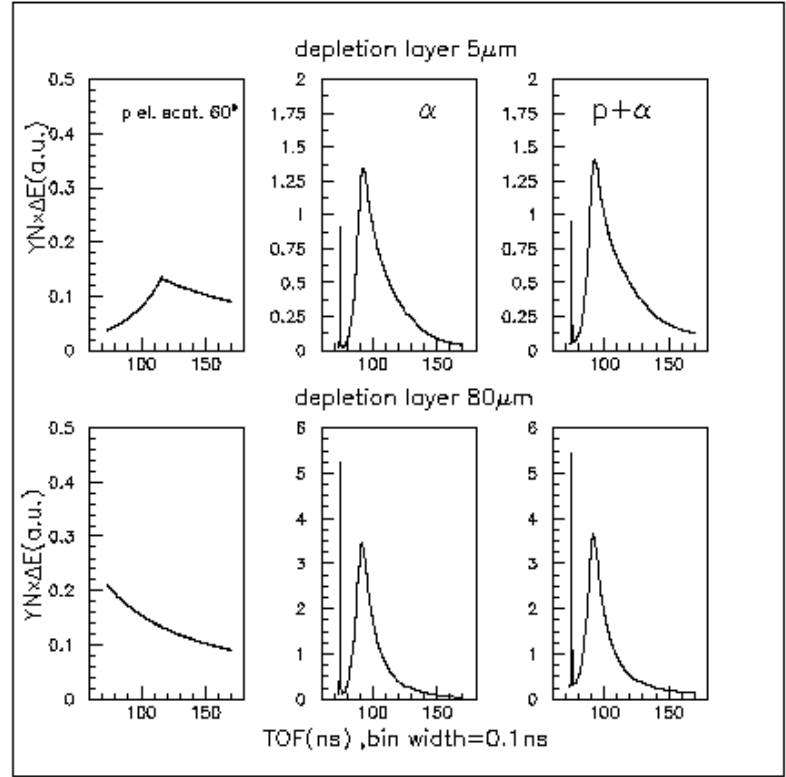

Fig. 3. Spectra of $p, \alpha,(p+\alpha)$ as modified by energy loss factors, for TOF with: $\mathrm{SiC} 5 \mu \mathrm{m}$ (top), $\mathrm{SiC} 80 \mu \mathrm{m}$ (bottom), respectively.

As first approximation, it is possible to assume these energy losses to be fully converted in e-hole pairs production. So, for each $\mathrm{p}$ and $\alpha$ particle, the associated charge signal would be proportional to $\Delta \mathrm{E}_{\mathrm{p}}, \Delta \mathrm{E}_{\alpha}$. Then the charge signals (yields) in each time bin are:

$$
\Delta \mathrm{Y}_{\mathrm{p}}=\mathrm{c} \mathrm{N} \underset{\mathrm{p}}{\mathrm{N}} \Delta \mathrm{E} \text {, and } \Delta \mathrm{Y}_{\alpha}=\mathrm{c} \mathrm{N} \mathrm{N}_{\alpha} \Delta \mathrm{E}_{\alpha}
$$

where $\mathrm{c}$ is a constant depending on readout circuit and, $\mathrm{N}_{\mathrm{p}}, \mathrm{N}_{\alpha}$ are the number of ions arriving into detector in time bin $\Delta \mathrm{T}$, as proton and alpha respectively.

Spectra $(p+\alpha)$ of figure 3 have been obtained by means of two equations (4), with $\mathrm{c}=1$, and represent the simulated responses of two $\mathrm{SiC}$ detectors.

We observe as the expected yields depend very strongly on ion energy losses in the active layer of detector so being possible be very different from the true values.

The method is based on the assumptions of:

(a)The active area of detectors to be equal/scaled because of collimating diaphragms and/or preventive calibration. The readout circuits of both detectors to have the same characteristics of gain and time resolution, $(\mathrm{c}=1)$. The contiguity, due to very small areal dimensions, and the long distance path of flight, to produce a very small change in the observation direction, assuring (within the statistical fluctuations) the same number of ions, $\mathrm{N}_{\mathrm{i} \text { on, }}$ to impinge on two $\mathrm{SiC}$ detectors.

(b) The charge measured by $\mathrm{SiC}$ device be proportional to product $\mathrm{N}_{\text {ion }} \times \Delta \mathrm{E}_{\text {ion }}$. 
Under the validity of (a) assumptions, it is expected, for each time $\mathrm{Ti}$, within the time bin, the number of ions arriving into two detectors to be the same.

Then: $\mathrm{N}_{\mathrm{p} 1}=\mathrm{N}_{\mathrm{p} 2}=\mathrm{N}_{\mathrm{p}}(\mathrm{Ti}), \mathrm{N}_{\alpha 1}=\mathrm{N}_{\alpha 2}=\mathrm{N}_{\alpha}(\mathrm{Ti})$ ( 5 )

for each time bin centered at $\mathrm{T} i$ :

$$
\text { det. } 1 \quad\left(\Delta \mathrm{Y}_{\mathrm{Ti}}\right)_{1}=\mathrm{N}_{\mathrm{p}} \times \Delta \mathrm{E}{ }_{\mathrm{p} 1}+\underset{\alpha}{\mathrm{N}} \times \Delta \mathrm{E}
$$

$$
\text { det. } 2\left(\Delta \mathrm{Y}_{\mathrm{Ti}}\right)_{2}=\mathrm{N}_{\mathrm{p}} \times \Delta \mathrm{E}_{\mathrm{p} 2}+\mathrm{N}_{\alpha} \times \Delta \mathrm{E}{ }_{\alpha 2}
$$

$\left(\Delta \mathrm{Y}_{\mathrm{Ti}}\right)_{1}$ and $\left(\Delta \mathrm{Y}_{\mathrm{Ti}}\right)_{2}$ are the measured total charges for each bin centered to $\mathrm{Ti}$, into detector 1 and detector 2 , respectively.

As it is shown in eqs. ( 6$)$ and ( 7$)$, first terms $\left(\Delta \mathrm{Y}_{\mathrm{Ti}}\right)_{1}$ and $\left(\Delta \mathrm{Y}_{\mathrm{Ti}}\right)_{2}$ can be measured; the right sides of two equations contain the energy losses of proton and alpha into detectors, which can be calculated by SRIM2008 code [22], so that, for each time $\mathrm{Ti}$, the true $\mathrm{N}_{\mathrm{p}}$ and $\mathrm{N}_{\alpha}$ can be determined.

Figure 4 shows the reconstructed spectra of proton, $\alpha$ particles and sum contributions.

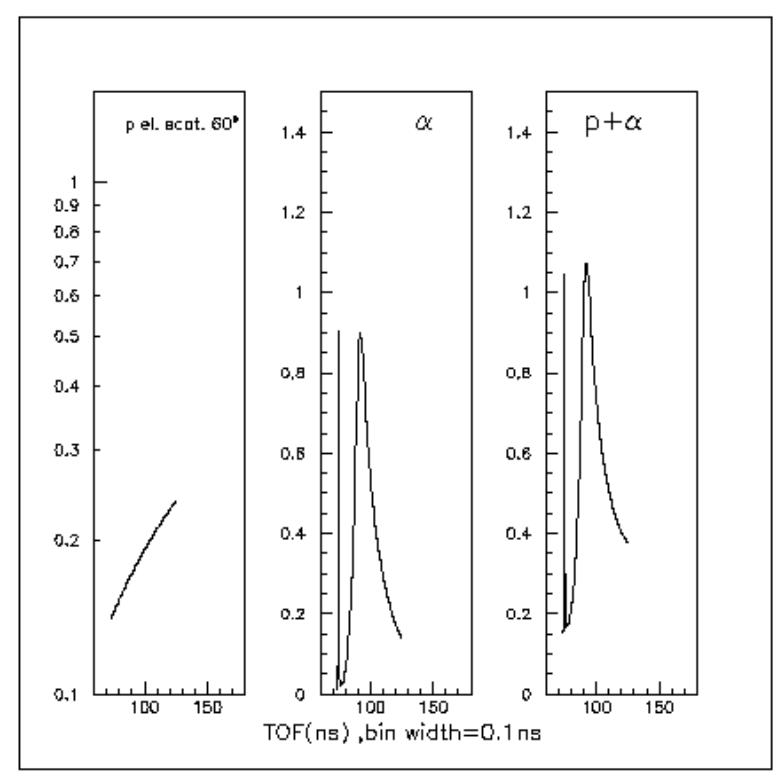

Fig.4. Reconstructed TOF spectra of proton, $\alpha$-particles and sum contributions.

\section{Discussion and summary}

From compared inspection of Figure 2 and Figure 4 it can be noticed how the described method works. In fact, Figure 2 (bottom) reports the true expected TOF-spectra for proton and $\alpha$-particles, whereas Figure 4 shows the same spectra reconstructed by means of eq.(6) and eq.(7) joint to simulated responses $(\mathrm{p}+\alpha)$ of $\mathrm{SiC}-5 \mu \mathrm{m}$ and $\mathrm{SiC}-$ $80 \mu \mathrm{m}$. The obtainable proton and alpha spectra are limited to TOF range where the corresponding energy losses for $\mathrm{p}$ and/or $\alpha$-particles are different in one, at least, detector.

Assumption (a) can be verified directly by setting in contiguous positions two $\mathrm{SiC}$ detectors of same depletion layer and readout characteristics.

To verify assumption (b), some dedicate experiment (where only one of ions to discriminate could be present) can be performed by using coupled detectors. For instance, $\mathrm{SiC}$ detector contiguous to an ion collector (IC) or $\mathrm{SiC}$ detector contiguous to pin-hole of a Thomson spectrometer equipped with CR-39 strips on focal plane. In that cases, $\mathrm{SiC}$ detector yield could give information on product $\mathrm{N}_{\text {ion }} \times \mathrm{N}_{\text {pair }}$, per each bin, whereas IC or CR-39 detectors, on true yield $\left(\mathrm{N}_{\text {ion }}\right)$. The discrimination has been treated only for proton and alpha particles but it can be extended to other cases as $(\mathrm{p}, \mathrm{d}),\left(\mathrm{d},{ }^{3} \mathrm{He}\right)$, etc and different depletion layer $\mathrm{SiC}$ detectors.

To summarize, in this paper the problem of Yielddependence on energy loss of ions in TOF-SiC technique, has been analyzed, showing the need to take into account for. On the assumption of few conditions, it has been found how the using of two contiguous $\mathrm{SiC}$ detectors of suitable characteristics and depleted layers, can allow to discriminate, in a large energy range, the contribution of competing light ions involved in plasmalaser induced nuclear reactions. However it has been shown the basic applicability of the method, without taking into account of limitations due to experimental sensitivities and statistical errors. Work is in progress in this perspective as well as to compare expected previsions with data of a real experiment.

\section{References}

1. V. S. Belyaev, A. P. Matafonov, and V. I. Vinogradov P. Krainov, S. Lisitsa, A. S. Roussetski, G. N. Ignatyev and V. P. Andrianov, Phys. Rev. E 72, 026406 (2005).

2. W. Bang, M. Barbui, A. Bonasera, H. J. Quevedo, G. Dyer, A. C. Bernstein, K. Hagel, K. Schmidt, E. Gaul, M. E. Donovan, F. Consoli, R. De Angelis, P. Andreoli, M. Barbarino, S. Kimura, M. Mazzocco, J. B. Natowitz, and T. Ditmire, Phys. Rev. E 88, 033108 (2013).

3. C. Labaune, C. Baccou, S. Depierreux, C. Goyon, G. Loisel, V. Yahia J. Rafelski, Nature Comms 4:2506 doi:10.1038/ncomms3506 (2013).

4. S Cavallaro, L Torrisi, M Cutroneo, J Krása and J Ullschmied, IOP Publishing Journal of Physics: Conference Series 508 (2014) 012023 doi:10.1088/ 17426596/508/1/012023.

5. D Margarone, A Picciotto, A Velyhan, J Krasa, M Kucharik, A Mangione, A Szydlowsky, A Malinowska, G Bertuccio, Y Shi, M Crivellari, J Ullschmied, P Bellutti and $\mathrm{G}$ Korn, Plasma Phys. and Contr. Fusion, Volume 57, Number 1 0741-3335/57/1/014030. Published 28 November 2014 • C 2015 IOP Publishing Ltd.

6. A. Picciotto, D. Margarone, A. Velyhan, P. Bellutti, J. Krasa, A. Szydlowsky, G. Bertuccio,Y. Shi, 
A. Mangione, J. Prokupek, A. Malinowska,E. Krousky, J. Ullschmied, L. Laska, M. Kucharik, and G. Korn,

Phys. Rev. X 4, 031030 (2014).

7. J. Krása, D. Klír, A. Velyhan, E. Krouský, M. Pfeifer, K. Řezáč, J. Cikhardt, K. Turek, J. Ullschmied and K. Jungwirth, (2014). High Power Laser Science and Engineering, 2, e19. doi:10.1017/hpl.2014.25.

8. D. Giulietti, P. Andreoli, D. Batani, A. Bonasera, D.G. Boutoux, F. Burgy, M. Cipriani, F. Consoli, G. Cristofari, R. De Angelis, G. Di Giorgio, J.E. Ducret, F. Ingenito, K. Jakubowska, C. Verona, G. Verona-Rinati,

Nucl. Instr. Methods B March 2017 DOI:

10.1016/j.nimb. 2017.03.076.

9. W. Bang, H. J. Quevedo, G. Dyer, J. Rougk, I. Kim, M. McCormick, A. C. Bernstein, and T. Ditmire,

Rev. Sci. Instrum. 83, 063504 (2012).

10. S. Cavallaro, L. Torrisi, $41^{\text {st }}$ EPS Conference on Plasma Physics Berlin 23-27 June2014P2.115. http://ocs.ciemat.es/EPS2014PAP/html/.

11. S. Cavallaro, Rev. Sci. Instrum.

86(3):036103 March 2015

12. A. Szydlowski, Rad. Meas. 36 (2003) 35-42.

13. C. Baccou,V. Yahia, S. Depierreux, C. Neuville, C. Goyon, F. Consoli, R. De Angelis, J. E. Ducret, G. Boutoux J. Rafelski, and C. Labaune, Rev. Sci. Instrum. 86, 083307 (2015).

14. A. Alejo, S. Kar, H. Ahmed, A. G. Krygier, D. Doria, R. Clarke, J. Fernandez, R. R. Freeman, J. Fuchs, A. Green, J. S. Green, D. Jung, A. Kleinschmidt, C. L. S. Lewis, J. T. Morrison, Z. Najmudin, H. Nakamura, G. Nersisyan, P. Norreys, M. Notley, M. Oliver, M. Roth J. A. Ruiz, L. Vassura, M. Zepf, and M. Borghesi,

Rev. Sci. Instrum. 85, 093303 (2014).

15. S. Cavallaro, L. Torrisi ,A. Amato ,L. Andò, M.Cutroneo, $39^{\text {th }}$ EPS Conference $\& 16^{\text {th }}$ Int. Congress on Plasma Physics. http://ocs.ciemat.es/ epsicpp2012/ pap/P4.121.pdf.

16. V. Togo, S. Balestra, S. Cecchini D. Di Ferdinando, M. Frutti, G. Giacomelli, M. Giorgini, A. Kuma, G. Mandrio, S. Manzoor, A. Margiotta, E. Medinaceli, L. Patrizi, V. Popa, M. Spurio, Nucl. Instr. Methods A 580 (2007) 58-61.

17. S. Cavallaro, D. Margarone, L.Torrisi

35th EPS Conference on Plasma Phys. Hersonissos, 9-13 June 2008 ECA Vol.32D, P-4.154 (2008).

18. A. Malinowska, M. Jaskóła, A. Korman, A. Szydłowski, and M. Kuk, Rev. Sci. Instrum. 85, 123505 (2014); doi: 10.1063/1.4903755.

19. M. Cutroneo, P. Musumeci, M. Zimbarnone, L. Torrisi, F. La Via, D. Margarone, A. Velyhan, J. Ullschmied,L. Calcagno, J. Mater. Res. 2012 DOI: 10.1557/jmr. 2012.211.

20. Giuseppe Bertuccio, Donatella Puglisi, Lorenzo Torrisi, Claudio Lanzieri, Appl. Surf. Sci. 272 (2013) 128-131 $381 \quad$ (2012) 012116 doi:10.1088/1742$6596 / 381 / 1 / 012116$.
21. C A Diget, H O U Fynbo, K L Laursen, K O Madsbøl, LJørgensen, O S Kirsebom,, and K Riisager Rutherford Centennial Conference on Nuclear Physics IOP Publishing Journal of Physics: Conference Series 381 (2012) 012116 doi:10.1088/17426596/381/1/012116 .093303 (2012).

22. J. F. Ziegler, J. P. Biersack, SRIM-2008 code, NEA-0919/06. 